\title{
Positive Solutions for Singular Boundary Value Problems of Coupled Systems of Nonlinear Differential Equations
}

\section{Ying He}

School of Mathematics and Statistics, Northeast Petroleum University, Daqing, China Email: heying65338406@163.com

Received 13 June 2014; revised 15 July 2014; accepted 26 July 2014

Copyright (C) 2014 by author and Scientific Research Publishing Inc.

This work is licensed under the Creative Commons Attribution International License (CC BY).

http://creativecommons.org/licenses/by/4.0/

(c) (i) Open Access

\section{Abstract}

We establish the existence of positive solutions for singular boundary value problems of coupled systems

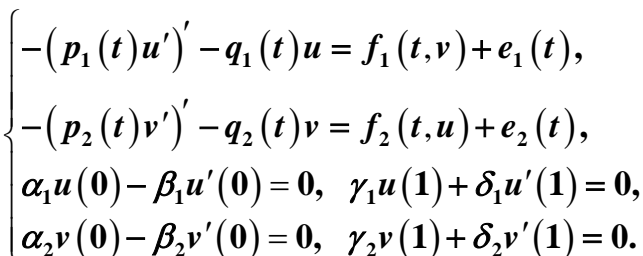

The proof relies on Schauder's fixed point theorem. Some recent results in the literature are generalized and improved.

\section{Keywords}

Positive Solutions, Second-Order Boundary Value Problems, Coupled Systems, Schauder's Fixed Point Theorem

\section{Introduction}

In this paper, we consider the existence of positive solutions for coupled singular system of second order ordinary differential equations

How to cite this paper: He, Y. (2014) Positive Solutions for Singular Boundary Value Problems of Coupled Systems of Nonlinear Differential Equations. Journal of Applied Mathematics and Physics, 2, 903-909. 


$$
\left\{\begin{array}{l}
-\left(p_{1}(t) u^{\prime}\right)^{\prime}-q_{1}(t) u=f_{1}(t, v)+e_{1}(t), \\
-\left(p_{2}(t) v^{\prime}\right)^{\prime}-q_{2}(t) v=f_{2}(t, u)+e_{2}(t), \\
\alpha_{1} u(0)-\beta_{1} u^{\prime}(0)=0, \quad \gamma_{1} u(1)+\delta_{1} u^{\prime}(1)=0, \\
\alpha_{2} v(0)-\beta_{2} v^{\prime}(0)=0, \quad \gamma_{2} v(1)+\delta_{2} v^{\prime}(1)=0 .
\end{array}\right.
$$

Throughout this paper, we always suppose that

$\left(S_{1}\right) \quad p_{i}(t) \in C^{1}([0,1], R), p_{i}(t)>0, \quad q_{i}(t) \in C([0,1], R), \quad q_{i}(t) \leq 0, \quad e_{i}(t) \in C([0,1], R) \alpha_{i}, \beta_{i}, \gamma_{i}, \delta_{i} \geq 0$, and $\beta_{i} \gamma_{i}+\alpha_{i} \gamma_{i}+\alpha_{i} \delta_{i}>0(i=1,2) . f_{1}, f_{2} \in C([0,1] \times(0,+\infty),(0,+\infty))$, and may be singular near the zero.

In recent years, singular boundary value problems to second ordinary differential equations have been studied extensively (see [1]-[3]). Some classical tools have been used in the literature to study the positive solutions for second order singular boundary value problems of a coupled system of differential equations. These classical methods include some fixed point theorems in cones for completely continuous operators and Schauder fixed point theorem, for example, see [4]-[6] and literatures therein. Motivated by the recent work on coupled systems of second-order differential equations, we consider the existence of singular boundary value problem. By means of the Schauder fixed point theorem, we study the existence of positive solutions of coupled system (1.1).

\section{Preliminary}

We consider the scalar equation

$$
-\left(p(t) u^{\prime}\right)^{\prime}-q(t) u=e(t)
$$

with boundary conditions

$$
\alpha u(0)-\beta u^{\prime}(0)=0, \quad \gamma u(1)+\delta u^{\prime}(1)=0,
$$

Suppose that $u$ is a positive solution of (2.1) and (2.2). Then

$$
u(t)=\int_{0}^{1} G(t, s) e(s) \mathrm{d} s .
$$

where $G(t, s)$ can be written by

$$
G(t, s):=\frac{1}{\omega} \begin{cases}m(t) n(s), & (t, s) \in Q_{1}, \\ m(s) n(t), & (t, s) \in Q_{2} .\end{cases}
$$

here $I=[0,1], Q=I \times I$ and $Q_{1}=\{(t, s) \in Q \mid 0 \leq t \leq s \leq 1\}, \quad Q_{2}=\{(t, s) \in Q \mid 0 \leq s \leq t \leq 1\}$.

Lemma 2.1. Suppose that $\left(S_{1}\right)$ holds, then the Green's function $G(t, s)$, defined by (2.3) possesses the following properties:

1): $m(t) \in C^{2}(I, R)$ is increasing and $m(t)>0, x \in(0,1]$.

2): $m(t) \in C^{2}(I, R)$ is decreasing and $n(t)>0, x \in[0,1)$.

3): $(\operatorname{Lm})(t) \equiv 0, m(0)=\beta, m^{\prime}(0)=\alpha$.

4): $(L n)(t) \equiv 0, n(1)=\delta, n^{\prime}(1)=-\gamma$.

5): $\omega$ is a positive constant. Moreover, $p(t)\left(m^{\prime}(t) n(t)-m(t) n^{\prime}(t)\right) \equiv \omega$.

6): $G(t, s)$ is continuous and symmetrical over $Q$.

7): $G(t, s)$ has continuously partial derivative over $Q_{1}, Q_{2}$.

8): For each fixed $s \in I, G(t, s)$ satisfies $L G(t, s)=0$ for $s \neq t, t \in I$. Moreover, $R_{1}(G)=R_{2}(G)=0$ for $s \in(0,1)$.

9): $G_{t}^{\prime}$ has discontinuous point of the first kind at $t=s$ and

$$
G_{t}^{\prime}(s+0, s)-G_{t}^{\prime}(s-0, s)=-\frac{1}{p(s)}, \quad s \in(0,1)
$$


We define the function $\gamma_{i}(t):[0,1] \rightarrow R$ by

$$
\gamma_{i}(t)=\int_{0}^{1} G_{i}(t, s) e_{i}(s) \mathrm{d} s, \quad i=1,2,
$$

which is the unique solution of

$$
\left\{\begin{array}{l}
-\left(p_{i}(t) u^{\prime}(t)\right)^{\prime}-q_{i}(t) u(t)=e_{i}(t), 0<t<1, \quad i=1,2 \\
\alpha_{i} u(0)-\beta_{i} u^{\prime}(0)=0 \\
\gamma_{i} u(1)+\delta_{i} u^{\prime}(1)=0 .
\end{array}\right.
$$

Following from Lemma 2.1 and $\left(S_{1}\right)$, it is easy to see that

$$
G_{i}(t, s)>0 \text {, for all }(t, s) \in[0,1] \times[0,1], \quad i=1,2
$$

Let us fix some notation to be used in the following: For a given function $h \in C[0,1]$, we denote the essential supremum and infimum by $h^{*}$ and $h_{*}$. if they exist. Let, $\gamma_{i^{*}}=\min _{t} \gamma_{i}(t), \gamma_{i}^{*}=\max _{t} \gamma_{i}(t)$.

\section{Main Results}

1) $\gamma_{1^{*}} \geq 0, \quad \gamma_{2^{*}} \geq 0$.

Theorem 3.1. We assume that there exists $b_{i} \geq 0, \hat{b}_{i} \geq 0$, and $0<\alpha_{i}<1$ such that

$$
\left(H_{1}\right) \quad \frac{\hat{b}_{i}(t)}{u^{\alpha_{i}}} \leq f_{i}(t, u) \leq \frac{b_{i}(t)}{u^{\alpha_{i}}} \text {, for all } u>0 \text {, a.e. } t \in(0,1), i=1,2
$$

If $\gamma_{1^{*}} \geq 0, \gamma_{2^{*}} \geq 0$, then there exists a positive solution of (1.1).

Proof A positive solution of (1.1) is just a fixed point of the completely continuous map $A(u, v)=\left(A_{1} u, A_{2} v\right): C[0,1] \times C[0,1] \rightarrow C[0,1] \times C[0,1]$ defined as

$$
\begin{aligned}
& \left(A_{1} u\right)(t):=\int_{0}^{1} G_{1}(t, s)\left[f_{1}(s, v(s))+e_{1}(s)\right] \mathrm{d} s=\int_{0}^{1} G_{1}(t, s) f_{1}(s, v(s)) \mathrm{d} s+\gamma_{1}(t) ; \\
& \left(A_{2} v\right)(t):=\int_{0}^{1} G_{2}(t, s)\left[f_{2}(s, u(s))+e_{2}(s)\right] \mathrm{d} s=\int_{0}^{1} G_{2}(t, s) f_{2}(s, u(s)) \mathrm{d} s+\gamma_{2}(t) ;
\end{aligned}
$$

By a direct application of Schauder's fixed point theorem, the proof is finished if we prove that A maps the closed convex set defined as

$$
K=\left\{(u, v) \in C[0,1] \times C[0,1]: r_{1} \leq u(t) \leq R_{1}, r_{2} \leq v(t) \leq R_{2} \text {, for all } t \in[0,1]\right\}
$$

into itself, where $R_{1}>r_{1}>0, R_{2}>r_{2}>0$ are positive constants to be fixed properly. For convenience, we introduce the following notations

$$
\beta_{i}(t)=\int_{0}^{1} G_{i}(t, s) b_{i}(s) \mathrm{d} s, \quad \hat{\beta}_{i}(t)=\int_{0}^{1} G_{i}(t, s) \hat{b}_{i}(s) \mathrm{d} s, \quad i=1,2 .
$$

Given $(u, v) \in K$, by the nonnegative sign of $G_{i}$ and $f_{i}, i=1,2$ we have

$$
\left(A_{1} u\right)(t)=\int_{0}^{1} G_{1}(t, s) f_{1}(s, v(s)) \mathrm{d} s+\gamma_{1}(t) \geq \int_{0}^{1} G_{1}(t, s) \frac{\hat{b}_{1}(s)}{v^{\alpha_{1}}(s)} \mathrm{d} s \geq \int_{0}^{1} G_{1}(t, s) \frac{\hat{b}_{1}(s)}{R_{2}^{\alpha_{1}}} \mathrm{~d} s \geq \hat{\beta}_{1^{*}} \frac{1}{R_{2}^{\alpha_{1}}}
$$

Note for every $(u, v) \in K$

$$
\left(A_{1} u\right)(t)=\int_{0}^{1} G_{1}(t, s) f_{1}(s, v(s)) \mathrm{d} s+\gamma_{1}(t) \leq \int_{0}^{1} G_{1}(t, s) \frac{b_{1}(s)}{v^{\alpha_{1}}(s)} \mathrm{d} s+\gamma_{1}^{*} \leq \int_{0}^{1} G_{1}(t, s) \frac{b_{1}(s)}{r_{2}^{\alpha_{1}}} \mathrm{~d} s+\gamma_{1}^{*} \leq \beta_{1}^{*} \frac{1}{r_{2}^{\alpha_{1}}}+\gamma_{1}^{*}
$$

Similarly, by the same strategy, we have

$$
\left(A_{2} v\right)(t)=\int_{0}^{1} G_{2}(t, s) f_{2}(s, u(s)) \mathrm{d} s+\gamma_{2}(t) \geq \int_{0}^{1} G_{2}(t, s) \frac{\hat{b}_{2}(s)}{u^{\alpha_{2}}(s)} \mathrm{d} s \geq \int_{0}^{1} G_{2}(t, s) \frac{\hat{b}_{2}(s)}{R_{1}^{\alpha_{2}}} \mathrm{~d} s \geq \hat{\beta}_{2^{*}} \frac{1}{R_{1}^{\alpha_{2}}}
$$




$$
\left(A_{2} v\right)(t)=\int_{0}^{1} G_{2}(t, s) f_{2}(s, u(s)) \mathrm{d} s+\gamma_{2}(t) \leq \int_{0}^{1} G_{2}(t, s) \frac{b_{2}(s)}{u^{\alpha_{2}}(s)} \mathrm{d} s+\gamma_{2}^{*} \leq \int_{0}^{1} G_{2}(t, s) \frac{b_{2}(s)}{r_{1}^{\alpha_{2}}} \mathrm{~d} s+\gamma_{2}^{*} \leq \beta_{2}^{*} \frac{1}{r_{1}^{\alpha_{2}}}+\gamma_{2}^{*}
$$

Thus $\left(A_{1} u, A_{2} v\right) \in K$ if $r_{1}, r_{2}, R_{1}, R_{2}$ are chosen so that

$$
\begin{aligned}
& \hat{\beta}_{1^{*}} \cdot \frac{1}{R_{2}^{\alpha_{1}}} \geq \gamma_{1}, \quad \beta_{1}^{*} \cdot \frac{1}{r_{2}^{\alpha_{1}}}+\gamma_{1}^{*} \leq R_{1} \\
& \hat{\beta}_{2^{*}} \cdot \frac{1}{R_{1}^{\alpha_{2}}} \geq \gamma_{2}, \quad \beta_{2}^{*} \cdot \frac{1}{r_{1}^{\alpha_{2}}}+\gamma_{2}^{*} \leq R_{1} .
\end{aligned}
$$
that

Note that $\hat{\beta}_{i^{*}}>0, \beta_{i^{*}}>0$ and taking $R=R_{1}=R_{2}, r=r_{1}=r_{2}, r=\frac{1}{R}$, it is sufficient to find $R>1$ such

$$
\begin{aligned}
& \hat{\beta}_{1^{*}} \cdot R^{1-\alpha_{1}} \geq 1, \quad \beta_{1}^{*} \cdot R^{\alpha_{1}}+\gamma_{1}^{*} \leq R \\
& \hat{\beta}_{2^{*}} \cdot R^{1-\alpha_{2}} \geq 1, \quad \beta_{2}^{*} \cdot R^{\alpha_{2}}+\gamma_{2}^{*} \leq R .
\end{aligned}
$$

and these inequalities hold for $R$ big enough because $\alpha_{i}<1$.

2) $\gamma_{1}^{*} \leq 0, \gamma_{2}^{*} \leq 0$.

The aim of this section is to show that the presence of a weak singular nonlinearity makes it possible to find positive solutions if $\gamma_{1}^{*} \leq 0, \quad \gamma_{2}^{*} \leq 0$.

Theorem 3.2. We assume that there exists $b_{i} \geq 0, \hat{b}_{i} \geq 0$, and $0<\alpha_{i}<1$ such that $\left(H_{1}\right)$ is satisfied. If $\gamma_{1}^{*} \leq 0, \quad \gamma_{2}^{*} \leq 0$ and

$$
\begin{aligned}
& r_{1^{*}} \geq\left[\alpha_{1} \alpha_{2} \cdot \frac{\hat{\beta}_{1^{*}}}{\left(\beta_{2}^{*}\right)^{\alpha_{1}}}\right]^{\frac{1}{1-\alpha_{1} \alpha_{2}}}\left(1-\frac{1}{\alpha_{1} \alpha_{2}}\right), \\
& r_{2^{*}} \geq\left[\alpha_{1} \alpha_{2} \cdot \frac{\hat{\beta}_{2^{*}}}{\left(\beta_{1}^{*}\right)^{\alpha_{2}}}\right]^{\frac{1}{1-\alpha_{1} \alpha_{2}}}\left(1-\frac{1}{\alpha_{1} \alpha_{2}}\right) .
\end{aligned}
$$

then there exists a positive solution of (1.1).

Proof In this case, to prove that $A: K \rightarrow K$ it is sufficient to find $0<r_{1}<R_{1}, \quad 0<r_{2}<R_{2}$ such that

$$
\begin{array}{ll}
\frac{\hat{\beta}_{1^{*}}}{R_{2}^{\alpha_{1}}}+\gamma_{1^{*}} \geq r_{1}, & \frac{\beta_{1}^{*}}{r_{2}^{\alpha_{1}}} \leq R_{1} \\
\frac{\hat{\beta}_{2^{*}}}{R_{1}^{\alpha_{2}}}+\gamma_{2^{*}} \geq r_{2}, & \frac{\beta_{2}^{*}}{r_{1}^{\alpha_{2}}} \leq R_{2}
\end{array}
$$

If we fix $R_{1}=\frac{\beta_{1}^{*}}{r_{2}^{\alpha_{1}}}, R_{2}=\frac{\beta_{2}^{*}}{r_{1}^{\alpha_{2}}}$, then the first inequality of (3.3) holds if $r_{2}$ satisfies

$$
\hat{\beta}_{2^{*}}\left(\beta_{1}^{*}\right)^{-\alpha_{2}} r_{2}^{\alpha_{1} \alpha_{2}}+\gamma_{2^{*}} \geq r_{2}
$$

or equivalently

$$
\gamma_{2^{*}} \geq g\left(r_{2}\right):=r_{2}-\frac{\hat{\beta}_{2^{*}}}{\left(\beta_{1}^{*}\right)^{\alpha_{2}}} r_{1}^{\alpha_{1} \alpha_{2}}
$$

The function $g\left(r_{2}\right)$ possesses a minimum at

$$
r_{20}:=\left[\alpha_{1} \alpha_{2} \cdot \frac{\hat{\beta}_{2^{*}}}{\left(\beta_{1}^{*}\right)^{\alpha_{2}}}\right]^{\frac{1}{1-\alpha_{1} \alpha_{2}}}
$$

Taking $r_{2}=r_{20}$, then (3.3) holds if 


$$
\gamma_{2^{*}} \geq g\left(r_{20}\right)=\left[\alpha_{1} \alpha_{2} \cdot \frac{\hat{\beta}_{2^{*}}}{\left(\beta_{1}^{*}\right)^{\alpha_{2}}}\right]^{\frac{1}{1-\alpha_{1} \alpha_{2}}}\left(1-\frac{1}{\alpha_{1} \alpha_{2}}\right)
$$

Similarly,

$$
\gamma_{1^{*}} \geq h\left(r_{1}\right):=r_{1}-\frac{\hat{\beta}_{1^{*}}}{\left(\beta_{2}^{*}\right)^{\alpha_{1}}} r_{1}^{\alpha_{1} \alpha_{2}}
$$

$h\left(r_{1}\right)$ possesses a minimum at

$$
\begin{gathered}
r_{10}:=\left[\alpha_{1} \alpha_{2} \cdot \frac{\hat{\beta}_{1^{*}}}{\left(\beta_{2}^{*}\right)^{\alpha_{1}}}\right]^{\frac{1}{1-\alpha_{1} \alpha_{2}}} \\
\gamma_{1^{*}} \geq\left[\alpha_{1} \alpha_{2} \cdot \frac{\hat{\beta}_{1^{*}}}{\left(\beta_{2}^{*}\right)^{\alpha_{1}}}\right]^{\frac{1}{1-\alpha_{1} \alpha_{2}}}\left(1-\frac{1}{\alpha_{1} \alpha_{2}}\right)
\end{gathered}
$$

Taking $r_{1}=r_{10}, r_{2}=r_{20}$, then the first inequalities in (3.2) and (3.3) hold if $\gamma_{1^{*}} \geq h\left(r_{1}\right)$ and $\gamma_{2^{*}} \geq g\left(r_{2}\right)$, which are just condition (3.1). The second inequalities hold directly from the choice of $R_{1}$ and $R_{2}$, so it remains to prove that $R_{1}=\frac{\beta_{1}^{*}}{r_{20}^{\alpha_{1}}}>r_{10}, R_{2}=\frac{\beta_{2}^{*}}{r_{10}^{\alpha_{2}}}>r_{20}$ This is easily verified through elementary computations:

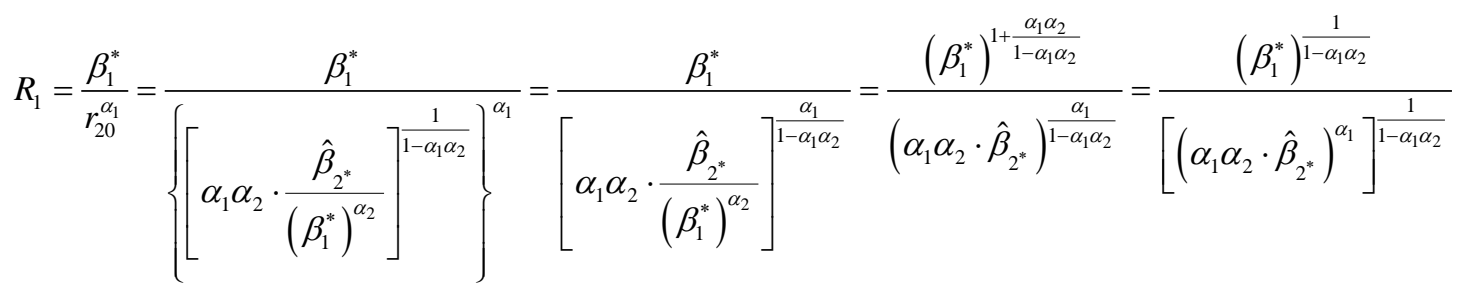

$$
\begin{aligned}
& =\left[\frac{\beta_{1}^{*}}{\left(\alpha_{1} \alpha_{2} \cdot \hat{\beta}_{2^{*}}\right)^{\alpha_{1}}}\right]^{\frac{1}{1-\alpha_{1} \alpha_{2}}}=\left[\frac{1}{\left(\alpha_{1} \alpha_{2}\right)^{\alpha_{1}}} \cdot \frac{\beta_{1}^{*}}{\left(\hat{\beta}_{2^{*}}\right)^{\alpha_{1}}}\right]^{\frac{1}{1-\alpha_{1} \alpha_{2}}}>\left[\alpha_{1} \alpha_{2} \cdot \frac{\hat{\beta}_{1^{*}}}{\left(\beta_{2}^{*}\right)^{\alpha_{1}}}\right]^{\frac{1}{1-\alpha_{1} \alpha_{2}}}=r_{10} \text {, }
\end{aligned}
$$

since $\hat{\beta}_{i^{*}} \leq \beta_{i}^{*}, i=1,2$ Similarly, we have $R_{2}>r_{20}$.

3) $\gamma_{1^{*}} \geq 0, \gamma_{2}^{*} \leq 0\left(\gamma_{1}^{*} \leq 0, \gamma_{2^{*}} \geq 0\right)$

Theorem 3.3. Assume that $\left(H_{1}\right)$ is satisfied. If $\gamma_{1^{*}} \geq 0, \gamma_{2}^{*} \leq 0$ and

$$
\gamma_{2^{*}} \geq r_{21}-\hat{\beta}_{2^{*}} \cdot \frac{r_{21}^{\alpha_{1} \alpha_{2}}}{\left(\beta_{1}^{*}+\gamma_{1}^{*} r_{21}^{\alpha_{1}}\right)^{\alpha_{2}}}
$$

where $0<r_{21}<+\infty$ is a unique positive solution of equation

$$
r_{2}^{1-\alpha_{1} \alpha_{2}}\left(\beta_{1}^{*}+\gamma_{1}^{*} \cdot r_{2}^{\alpha_{1}}\right)^{1+\alpha_{2}}=\alpha_{1} \alpha_{2} \beta_{1}^{*} \hat{\beta}_{2^{*}}
$$

then there exists a positive solution of (1.1).

Proof We follow the same strategy and notation as in the proof of ahead theorem. In this case, to prove that $A: K \rightarrow K$, it is sufficient to find $r_{1}<R_{1}, r_{2}<R_{2}$ such that

$$
\frac{\hat{\beta}_{1^{*}}}{R_{2}^{\alpha_{1}}} \geq r_{1}, \quad \frac{\beta_{2}^{*}}{r_{1}^{\alpha_{2}}} \leq R_{2}
$$




$$
\frac{\hat{\beta}_{2^{*}}}{R_{1}^{\alpha_{2}}}+\gamma_{2^{*}} \geq r_{2}, \quad \frac{\beta_{1}^{*}}{r_{2}^{\alpha_{1}}}+\gamma_{1}^{*} \leq R_{1}
$$

If we fix $R_{2}=\frac{\beta_{2}^{*}}{r_{1}^{\alpha_{2}}}$, then the first inequality of (3.6) holds if $r_{1}$ satisfies

$$
\frac{\hat{\beta}_{1^{*}}}{\left(\beta_{2}^{*}\right)^{\alpha_{1}}} \cdot r_{1}^{\alpha_{1} \alpha_{2}} \geq r_{1}
$$

or equivalently

$$
0<r_{1} \leq\left[\frac{\hat{\beta}_{1^{*}}}{\left(\beta_{2}^{*}\right)^{\alpha_{1}}}\right]^{\frac{1}{1-\alpha_{1} \alpha_{2}}}
$$

If we chose $r_{1}>0$ small enough, then (3.9) holds, and $R_{2}$ is big enough.

If we fix $R_{1}=\frac{\beta_{1}^{*}}{r_{2}^{\alpha_{1}}}+\gamma_{1}^{*}$ then the first inequality of (3.7) holds if $r_{2}$ satisfies

$$
\gamma_{2^{*}} \geq r_{2}-\frac{\hat{\beta}_{2^{*}}}{R_{1}^{\alpha_{2}}}=r_{2}-\hat{\beta}_{2^{*}} \cdot \frac{1}{\left(\frac{\beta_{1}^{*}}{r_{2}^{\alpha_{1}}}+\gamma_{1}^{*}\right)^{\alpha_{2}}}=r_{2}-\hat{\beta}_{2^{*}} \cdot \frac{1}{\left(\frac{\beta_{1}^{*}+\gamma_{1}^{*} \cdot r_{2}^{\alpha_{1}}}{r_{2}^{\alpha_{1}}}\right)^{\alpha_{2}}}=r_{2}-\hat{\beta}_{2^{*}} \cdot \frac{r_{2}^{\alpha_{1} \alpha_{2}}}{\left(\beta_{1}^{*}+\gamma_{1}^{*} \cdot r_{2}^{\alpha_{1}}\right)^{\alpha_{2}}},
$$

or equivalently

$$
\gamma_{2^{*}} \geq f\left(r_{2}\right):=r_{2}-\hat{\beta}_{2^{*}} \cdot \frac{r_{2}^{\alpha_{1} \alpha_{2}}}{\left(\beta_{1}^{*}+\gamma_{1}^{*} \cdot r_{2}^{\alpha_{1}}\right)^{\alpha_{2}}}
$$

According to

$$
\begin{aligned}
f^{\prime}\left(r_{2}\right) & =1-\hat{\beta}_{2^{*}} \cdot \frac{1}{\left(\beta_{1}^{*}+\gamma_{1}^{*} \cdot r_{2}^{\alpha_{1}}\right)^{2 \alpha_{2}}}\left[\alpha_{1} \alpha_{2} r_{2}^{\alpha_{1} \alpha_{2}-1}\left(\beta_{1}^{*}+\gamma_{1}^{*} \cdot r_{2}^{\alpha_{1}}\right)^{\alpha_{2}}-r_{2}^{\alpha_{1} \alpha_{2}} \alpha_{2}\left(\beta_{1}^{*}+\gamma_{1}^{*} \cdot r_{2}^{\alpha_{1}}\right)^{\alpha_{2}-1} \alpha_{1} \gamma_{1}^{*} r_{2}^{\alpha_{1}-1}\right] \\
& =1-\frac{\hat{\beta}_{2^{*}} \alpha_{1} \alpha_{2} r_{2}^{\alpha_{1} \alpha_{2}-1}}{\left(\beta_{1}^{*}+\gamma_{1}^{*} \cdot r_{2}^{\alpha_{1}}\right)^{\alpha_{2}}}\left[1-\frac{r_{2}^{\alpha_{1}} \gamma_{1}^{*}}{\beta_{1}^{*}+\gamma_{1}^{*} \cdot r_{2}^{\alpha_{1}}}\right]=1-\alpha_{1} \alpha_{2} \beta_{1}^{*} \hat{\beta}_{2^{*}} r_{2}^{\alpha_{1} \alpha_{2}-1}\left(\beta_{1}^{*}+\gamma_{1}^{*} \cdot r_{2}^{\alpha_{1}}\right)^{-1-\alpha_{2}},
\end{aligned}
$$

we have $f^{\prime}(0)=-\infty, f^{\prime}(+\infty)=1$, then there exists $r_{21}$ such that $f^{\prime}\left(r_{21}\right)=0$, and

$$
\begin{aligned}
f^{\prime \prime}\left(r_{2}\right)= & -\left[\alpha_{1} \alpha_{2} \beta_{1}^{*} \hat{\beta}_{2^{*}}\left(\alpha_{1} \alpha_{2}-1\right) r_{2}^{\alpha_{1} \alpha_{2}-2}\left(\beta_{1}^{*}+\gamma_{1}^{*} \cdot r_{2}^{\alpha_{1}}\right)^{-1-\alpha_{2}}\right. \\
& \left.+\alpha_{1} \alpha_{2} \beta_{1}^{*} \hat{\beta}_{2^{*}} r_{2}^{\alpha_{1} \alpha_{2}-1}\left(-1-\alpha_{2}\right)\left(\beta_{1}^{*}+\gamma_{1}^{*} \cdot r_{2}^{\alpha_{1}}\right)^{-2-\alpha_{2}} \gamma_{1}^{*} \alpha_{1} r_{2}^{\alpha_{1}-1}\right]>0 .
\end{aligned}
$$

Then the function $f\left(r_{2}\right)$ possesses a minimum at $r_{21}$, i.e., $f\left(r_{21}\right)=\min _{r_{2} \in(0,+\infty)} f\left(r_{2}\right)$. Note $f^{\prime}\left(r_{21}\right)=0$ then we have

$$
1-\alpha_{1} \alpha_{2} \beta_{1}^{*} \hat{\beta}_{2^{*}} r_{21}^{\alpha_{1} \alpha_{2}-1}\left(\beta_{1}^{*}+\gamma_{1}^{*} \cdot r_{21}^{\alpha_{1}}\right)^{-1-\alpha_{2}}=0
$$

or equivalently

$$
r_{21}^{1-\alpha_{1} \alpha_{2}}\left(\beta_{1}^{*}+\gamma_{1}^{*} \cdot r_{21}^{\alpha_{1}}\right)^{1+\alpha_{2}}=\alpha_{1} \alpha_{2} \beta_{1}^{*} \hat{\beta}_{2^{*}}
$$

Taking $r_{2}=r_{21}$, then the first inequality in (3.7) holds if $\gamma_{2^{*}} \geq f\left(r_{21}\right)$, which is just condition (3.4). The second inequalities hold directly by the choice of $R_{1}$, and it would remain to prove that $r_{21}<R_{2}$ and $r_{10}<R_{1}$. These inequalities hold for $R_{2}$ big enough and $r_{1}$ small enough. 
Remark 1. In theorem 3.3 the right-hand side of condition (3.4) always negative, this is equivalent to proof that $f\left(r_{21}\right)<0$. This is obviously established through the proof of Theorem 3.3.

Similarly, we have the following theorem.

Theorem 3.4. Assume $\left(H_{1}\right)$ is satisfied. If $\gamma_{1}^{*} \leq 0, \gamma_{2^{*}} \geq 0$ and

$$
\gamma_{1^{*}} \geq r_{11}-\hat{\beta}_{1^{*}} \cdot \frac{r_{11}^{\alpha_{1} \alpha_{2}}}{\left(\beta_{2}^{*}+\gamma_{2}^{*} r_{11}^{\alpha_{2}}\right)^{\alpha_{1}}},
$$

where $0<r_{11}<+\infty$ is a unique positive solution of the equation

$$
r_{1}^{1-\alpha_{1} \alpha_{2}}\left(\beta_{2}^{*}+\gamma_{2}^{*} r_{1}^{\alpha_{2}}\right)^{1+\alpha_{1}}=\alpha_{1} \alpha_{2} \beta_{2}^{*} \hat{\beta}_{1^{*}},
$$

then there exists a positive solution of (1.1).

\section{Funding}

Project supported by Heilongjiang province education department natural science research item, China (12541076).

\section{References}

[1] Jiang, D., Chu, J. and Zhang, M. (2005) Multiplicity of Positive Periodic Solutions to Superlinear Repulsive Singular Equations. Journal of Differential Equations, 211, 282-302. http://dx.doi.org/10.1016/j.jde.2004.10.031

[2] Torres, P.J. (2007) Weak Singularities May Help Periodic Solutions to Exist. Journal of Differential Equations, 232, 277-284. http://dx.doi.org/10.1016/j.jde.2006.08.006

[3] Chu, J. and Torres, P.J. (2007) Applications of Schauder's Fixed Point Theorem to Singular Differential Equations. Bulletin of the London Mathematical Society, 39, 653-660. http://dx.doi.org/10.1112/blms/bdm040

[4] Lv, H.Y., Yu, H.M. and Liu, Y.S. (2005) Positive Solutions for Singular Boundary Value Problems of a Coupled System of Differential Equations. Journal of Mathematical Analysis and Applications, 302, 14-29. http://dx.doi.org/10.1016/j.jmaa.2004.08.003

[5] Agarwal, R.P. and O’Regan, D. (2000) Multiple Solutions for a Coupled System of Boundary Value Problems. Dynamics of Continuous, Discrete and Impulsive Systems, 7, 97-106.

[6] Agarwal, R.P. and O’Regan, D. (1998) A Coupled System of Boundary Value Problems. Journal of Mathematical Analysis and Applications, 69, 381-385. http://dx.doi.org/10.1080/00036819808840668 
Scientific Research Publishing (SCIRP) is one of the largest Open Access journal publishers. It is currently publishing more than 200 open access, online, peer-reviewed journals covering a wide range of academic disciplines. SCIRP serves the worldwide academic communities and contributes to the progress and application of science with its publication.

Other selected journals from SCIRP are listed as below. Submit your manuscript to us via either submit@scirp.org or Online Submission Portal.
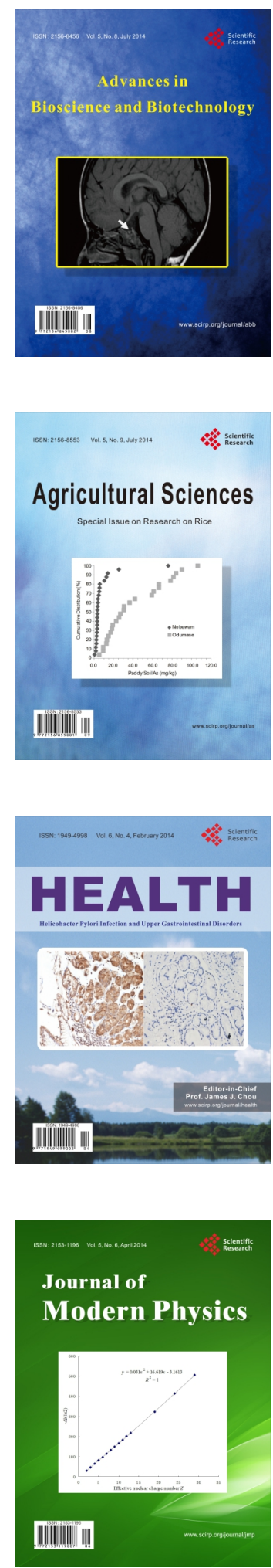
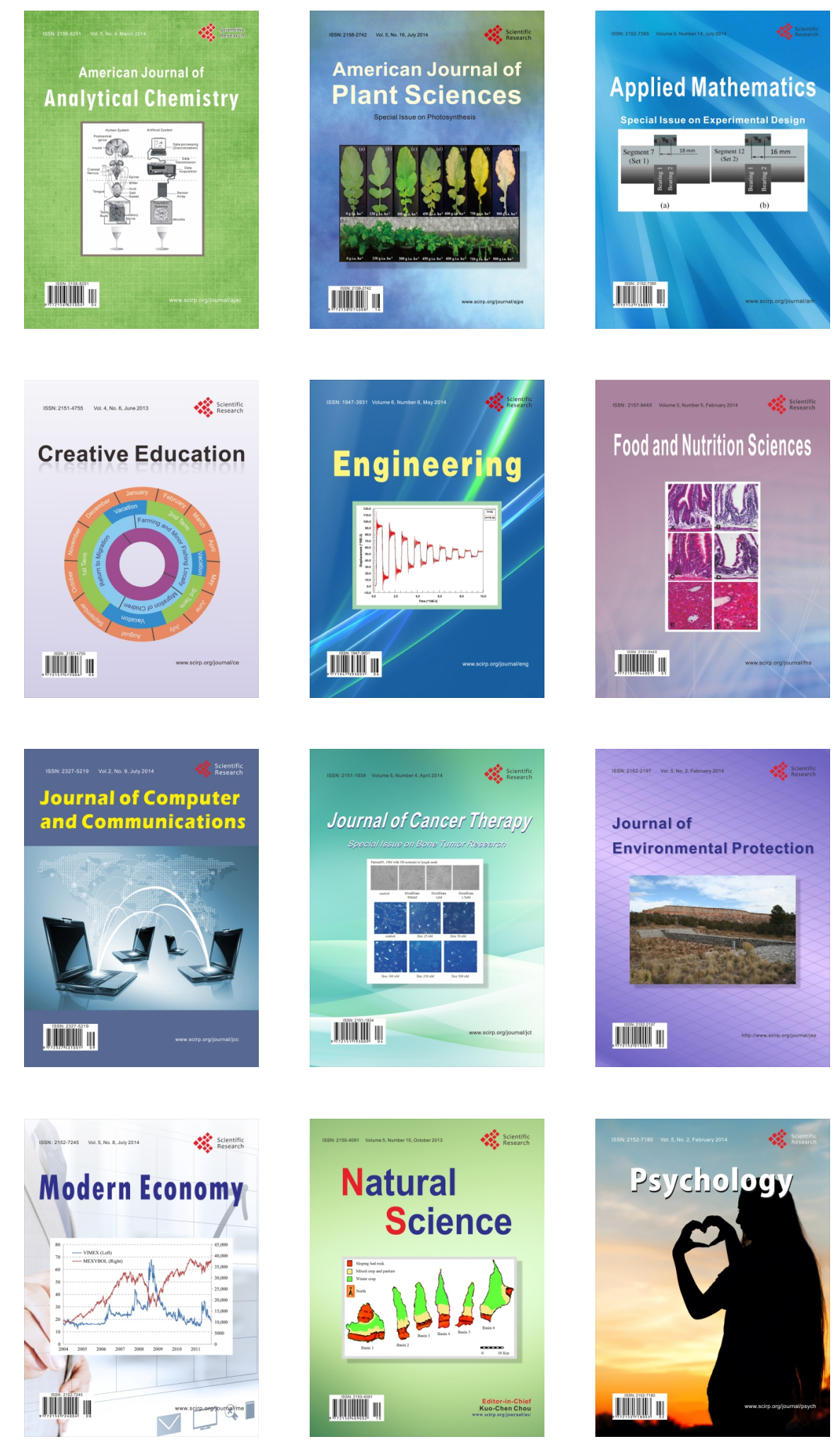